\title{
OPTIMAL CONSTANT IN A NEW ESTIMATE FOR THE DEGREE
}

\author{
By \\ HOAI-MinH NGUYEN
}

\begin{abstract}
In this paper, we prove the estimate

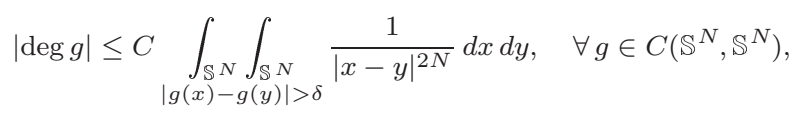

for every $\delta \in\left(0, \ell_{N}\right)$, where $C=C(N)$ is a positive constant depending only on $N$ and $\ell_{N}=\sqrt{2+2 /(N+1)}$. We show that the constant $\ell_{N}$ in this estimate is optimal. We also present a class of maps from $\mathbb{S}^{N}$ into $\mathbb{S}^{N}$, strictly larger than $C\left(\mathbb{S}^{N}, \mathbb{S}^{N}\right)$, on which we can define the notion of degree and for which the previous inequality still holds.
\end{abstract}

\section{Introduction}

In [3], the authors proved that

$$
|\operatorname{deg} g| \leq C \int_{\mathbb{S}^{N}} \int_{\mathbb{S}^{N}} \frac{1}{|g(x)-g(y)|>\delta}|y|^{2 N} d x d y, \quad \forall g \in C\left(\mathbb{S}^{N}, \mathbb{S}^{N}\right),
$$

for each $0<\delta<\sqrt{2}$, where $C=C(\delta, N)$ is a positive constant depending only on $N$ and $\delta$.

Here $|x|$ denotes Euclidean norm of $x$ for $x \in \mathbb{R}^{N+1}$.

Estimate (1.1) was initially suggested by J. Bourgain, H. Brezis, and P. Mironescu in [1]. This was proved in [2] in the case $N=1$ and $\delta$ sufficiently small. It is natural to ask whether (1.1) holds for every $0<\delta<2$. In this paper, we show that (1.1) holds if and only if $0<\delta<\sqrt{2+2 /(N+1)}$; moreover, the constant $C$ in this assertion can be chosen independently of $\delta$.

More precisely, set

$$
\ell_{N}=\sqrt{2+\frac{2}{N+1}}
$$

Our main result is the following 
Theorem 1. There exists a positive constant $C=C(N)$, depending only on $N$, such that for every $g \in C\left(\mathbb{S}^{N}, \mathbb{S}^{N}\right)$ and every $\delta \in\left(0, \ell_{N}\right)$,

$$
|\operatorname{deg} g| \leq C \int_{\mathbb{S}^{N}} \int_{\mathbb{S}^{N}} \frac{1}{|g(x)-g(y)|>\delta} \frac{1}{|x-y|^{2 N}} d x d y .
$$

Furthermore, there exists a sequence $\left\{g_{k}\right\}_{k \in \mathbb{N}} \subset C\left(\mathbb{S}^{N}, \mathbb{S}^{N}\right)$ such that

$$
\operatorname{deg} g_{k}=1, \quad \forall k \geq 1,
$$

and

$$
\lim _{k \rightarrow \infty} \int_{\substack{\mathbb{S}^{N} \\\left|g_{k}(x)-g_{k}(y)\right|>\ell_{N}}} \int_{\mathbb{S}^{N}} \frac{1}{|x-y|^{2 N}} d x d y=0 .
$$

The following corollary is a consequence of (1.3) (in fact, it is equivalent to $(1.3))$.

Corollary 1. There exists a positive constant $C=C(N)$, depending only on $N$, such that for every $g \in C\left(\mathbb{S}^{N}, \mathbb{S}^{N}\right)$,

$$
|\operatorname{deg} g| \leq C \int_{\mathbb{S}^{N}} \int_{\mathbb{S}^{N}} \frac{1}{|g(x)-g(y)| \geq \ell_{N}}|y|^{2 N} d x d y .
$$

In fact, from (1.3), we have

$$
|\operatorname{deg} g| \leq C_{N} \int_{\mathbb{S}^{N}} \int_{\mathbb{S}^{N}} \frac{1}{|g(x)-g(y)|>\delta} d x d y, \quad \forall 0<\delta<\ell_{N}
$$

for some positive constant $C=C(N)$ depending only on $N$. Thus, applying Lebesgue's dominated convergence theorem, one gets

$$
|\operatorname{deg} g| \leq C \liminf _{\delta \nearrow \ell_{N}} \int_{\substack{\mathbb{S}^{N} \\|g(x)-g(y)|>\delta}} \int_{\mathbb{S}^{N}} \frac{1}{|x-y|^{2 N}} d x d y=C \int_{\mathbb{S}^{N}} \int_{\mathbb{S}^{N}} \frac{1}{|g(x)-g(y)| \geq \ell_{N}} \frac{1}{|x-y|^{2 N}} d x d y .
$$

More generally, we show that if $g \in L^{\infty}\left(\mathbb{S}^{N}, \mathbb{S}^{N}\right)$ satisfies

$$
\int_{\mathbb{S}^{N}} \int_{\mathbb{S}^{N}} \frac{1}{|g(x)-g(y)|>\delta} d x d y<+\infty
$$

for some $0<\delta<\ell_{N}$, then we can define the degree of $g$ as in [5] and, moreover, assertion (1.3) holds, i.e.,

$$
|\operatorname{deg} g| \leq C \int_{\mathbb{S}^{N}} \int_{\mathbb{S}^{N}} \frac{1}{|g(x)-g(y)|>\delta} d x d y
$$


for some positive constant $C=C(N)$.

The proof of assertion (1.3) develops the idea used in [3]. To prove it, we first follow the strategy used in [3]. Then we establish a "generalized" version of [3, Lemma 2.1] (Lemma 6). We note that [3, Lemma 2.1] plays an important role in the proof of (1.1) (see [3]) and that it only holds for $\delta<\sqrt{2}$. Lemma 6 can be seen as a "continuous" version of Corollary 4 which is a consequence of Lemma 5. We use Corollary 3 in the proof of Lemma 5. Corollary 3 is a direct consequence of Lemma 3, dealing with a nice geometric property of $\mathbb{S}^{N}$, and Carathéodory's theorem (see [6]).

The idea of the construction of the sequence $\left(g_{k}\right)_{k \in \mathbb{N}}$ satisfying (1.4) and (1.5) is to construct continuous maps $g_{k}(k \in \mathbb{N})$, homotopic to the identity map, whose images are more and more concentrated on the set $\left\{A_{i}: 1 \leq i \leq N+2\right\}$, where $A_{1}, \ldots, A_{N+2}$, which lie on $\mathbb{S}^{N}$, are vertices of a $(N+2)$-vertex regular polyhedron.

The plan of the paper is as follows.

Section 2 discusses a weak version of assertion (1.3) of Theorem 1. In this section, we show that there exists a positive constant $C=C(N, \delta)$, depending only on $N$ and $\delta$, such that

$$
|\operatorname{deg} g| \leq C \int_{\mathbb{S}^{N}} \int_{\mathbb{S}^{N}} \frac{1}{|g(x)-g(y)|>\delta} d x d y, \quad \forall g \in C\left(\mathbb{S}^{N}, \mathbb{S}^{N}\right) .
$$

In Section 3, we construct a sequence $\left\{g_{k}\right\}_{k \in \mathbb{N}} \subset C\left(\mathbb{S}^{N}, \mathbb{S}^{N}\right)$ satisfying (1.4) and (1.5).

In Section 4, we prove that the constant $\mathrm{C}(=C(N, \delta))$ in $(1.8)$ can be chosen independently of $\delta$. This completes the proof of Theorem 1 .

Finally, in Section 5, we show that if $g \in L^{\infty}\left(\mathbb{S}^{N}, \mathbb{S}^{N}\right)$ satisfies (1.6) for some $0<\delta<\ell_{N}$, then $\operatorname{deg} g$ is well-defined in a manner similar to the one as in [5]. Inequality (1.7) is also proved in this section.

\section{Proof of assertion (1.8)}

2.1 A useful lemma. We begin this section with the following lemma, whose consequence (Corollary 2) is useful in the proof of (1.8).

Lemma 1. Let $g \in L^{\infty}\left(\mathbb{S}^{N}, \mathbb{S}^{N}\right)$, D be a measurable subset of $\mathbb{S}^{N}$ with $|\mathcal{D}|>0$, and $\lambda: \mathcal{D} \rightarrow[0,+\infty)$ be a measurable function. Assume that there exists a constant $\beta \geq 1$ such that $1 / \beta \leq \lambda(s) \leq \beta$ for every $s \in \mathcal{D}$ and

$$
\left|f_{\mathcal{D}} \lambda(s) g(s) d s\right| \leq \frac{1}{8 \beta(N+2)}\left[\ell_{N}^{2}-\left(\frac{\ell_{N}+\delta}{2}\right)^{2}\right] .
$$




\section{Then}

$$
\operatorname{meas}(\{(\xi, \eta) \in \mathcal{D} \times \mathcal{D}:|g(\xi)-g(\eta)|>\delta\}) \geq C|\mathcal{D}|^{2}
$$

for some $C=C(\delta, \beta, N)$.

Henceforth, $|\mathcal{D}|$ denotes the Lebesgue measure of $\mathcal{D}$ on $\mathbb{S}^{N}$ for any $\mathcal{D}$ measurable subset of $\mathbb{S}^{N}$.

\section{Proof. Set}

$$
\left\{\begin{array}{l}
\alpha=\frac{1}{8 \beta(N+2)}\left[\ell_{N}^{2}-\left(\frac{\ell_{N}+\delta}{2}\right)^{2}\right], \\
\varepsilon_{1}=\frac{1}{16 \beta^{2}(N+2)}\left[\ell_{N}^{2}-\left(\frac{\ell_{N}+\delta}{2}\right)^{2}\right], \\
\varepsilon=\frac{1}{16(N+2)}\left[\ell_{N}^{2}-\left(\frac{\ell_{N}+\delta}{2}\right)^{2}\right] .
\end{array}\right.
$$

Then

$$
\left|f_{\mathcal{D}} g(s) d s\right| \leq \alpha
$$

and

$$
\varepsilon=\beta^{2} \varepsilon_{1}=\beta \alpha / 2
$$

Let $\Omega_{1}, \ldots, \Omega_{k_{1}}$, be measurable subsets of $\mathbb{S}^{N}$ such that $\mathbb{S}^{N}=\bigcup_{i=1}^{k_{1}} \Omega_{i}$, $\Omega_{i} \cap \Omega_{j}=\emptyset$ for every $1 \leq i \neq j \leq k_{1}$, and $\operatorname{diam}\left(\Omega_{i}\right) \leq \varepsilon_{1} / 2$ for every $1 \leq i \leq k_{1}$. Set

$$
\mathcal{D}_{i}=g^{-1}\left(\Omega_{i}\right) \cap \mathcal{D}, \quad \forall 1 \leq i \leq k_{1}
$$

Define

$$
J=\left\{1 \leq i \leq k_{1}:\left|\mathcal{D}_{i}\right| \geq \frac{1}{m_{1}}|\mathcal{D}|\right\}
$$

where

$$
m_{1}=32(N+2) \beta^{2} k_{1} /\left[\ell_{N}^{2}-\left(\frac{\ell_{N}+\delta}{2}\right)^{2}\right]
$$

Without loss of generality, we assume that $J=\{1, \ldots, k\}$ for some $k \leq k_{1}$. Since $\left\{\mathcal{D}_{i}\right\}_{i=1}^{k_{1}}$ is a partition of $\mathcal{D}$,

$$
f_{\mathcal{D}} \lambda(s) g(s) d s=\frac{1}{|\mathcal{D}|} \sum_{i=1}^{k} \int_{\mathcal{D}_{i}} \lambda(s) g(s) d s+\frac{1}{|\mathcal{D}|} \sum_{i=k+1}^{k_{1}} \int_{\mathcal{D}_{i}} \lambda(s) g(s) d s .
$$


This implies

$$
\left|\frac{1}{|\mathcal{D}|} \sum_{i=1}^{k} \int_{\mathcal{D}_{i}} \lambda(s) g(s) d s\right| \leq\left|f_{\mathcal{D}} \lambda(s) g(s) d s\right|+\left|\frac{1}{|\mathcal{D}|} \sum_{i=k+1}^{k_{1}} \int_{\mathcal{D}_{i}} \lambda(s) g(s) d s\right| .
$$

Thus, since $i \notin J$ for all $k<i \leq k_{1}$ and $|\lambda(s)| \leq \beta$ for $s \in \mathcal{D}$, it follows from (2.2) and (2.5) that

$$
\left|\frac{1}{|\mathcal{D}|} \sum_{i=1}^{k} \int_{\mathcal{D}_{i}} \lambda(s) g(s) d s\right| \leq \alpha+\beta k_{1} / m_{1} .
$$

For each $i$, fix $A_{i} \in \Omega_{i}$. Then

$$
\begin{aligned}
\left|\frac{1}{|\mathcal{D}|} \sum_{i=1}^{k} \int_{\mathcal{D}_{i}} \lambda(s) d s A_{i}\right| \leq \\
\qquad\left|\frac{1}{|\mathcal{D}|} \sum_{i=1}^{k} \int_{\mathcal{D}_{i}} \lambda(s)\left(A_{i}-g(s)\right) d s\right|+\left|\frac{1}{|\mathcal{D}|} \sum_{i=1}^{k} \int_{\mathcal{D}_{i}} \lambda(s) g(s) d s\right| .
\end{aligned}
$$

Since $\operatorname{diam}\left(\Omega_{i}\right) \leq \varepsilon_{1} / 2$ and $A_{i} \in \Omega_{i}$, it follows from (2.4) that $\left|A_{i}-g(s)\right| \leq \varepsilon_{1}$ for all $s \in \mathcal{D}_{i}$. Thus, since $|\lambda(s)| \leq \beta$ for all $s \in \mathcal{D}$, we deduce from (2.7) and (2.8) that

$$
\left|\sum_{i=1}^{k} \int_{\mathcal{D}_{i}} \lambda(s) d s A_{i}\right| \leq\left(\beta \varepsilon_{1}+\alpha+\beta k_{1} / m_{1}\right)|\mathcal{D}|,
$$

which shows that $(\operatorname{since} \lambda(s) \geq 1 / \beta$ for all $s \in \mathcal{D})$

$$
\left|\sum_{i=1}^{k} c_{i} A_{i}\right| \leq\left(\beta \varepsilon_{1}+\alpha+\beta k_{1} / m_{1}\right) \frac{\beta|\mathcal{D}|}{\sum_{i=1}^{k}\left|\mathcal{D}_{i}\right|},
$$

where

$$
c_{i}=\left(\int_{\mathcal{D}_{i}} \lambda(s) d s\right) /\left(\sum_{i=1}^{k} \int_{\mathcal{D}_{i}} \lambda(s) d s\right) .
$$

Consequently,

$$
\left|\sum_{i=1}^{k} c_{i} A_{i}\right| \leq \frac{\beta\left(\beta \varepsilon_{1}+\alpha+\beta k_{1} / m_{1}\right)}{1-k_{1} / m_{1}} .
$$

On the other hand, since $\varepsilon \leq 1 / 4$ and $\beta \geq 1$, it follows from (2.3) and (2.6) that

$$
\frac{\beta\left(\beta \varepsilon_{1}+\alpha+\beta k_{1} / m_{1}\right)}{1-k_{1} / m_{1}}=\frac{\varepsilon+2 \varepsilon+\varepsilon / 2}{1-\varepsilon /\left(2 \beta^{2}\right)} \leq \frac{\varepsilon+2 \varepsilon+\varepsilon / 2}{1-1 / 8}=4 \varepsilon,
$$

which yields

$$
\left|\sum_{i=1}^{k} c_{i} A_{i}\right| \leq 4 \varepsilon
$$


Set

$$
A=\sum_{i=1}^{k} c_{i} A_{i}
$$

Then

$$
|A| \leq 4 \varepsilon=\frac{1}{4(N+2)}\left[\ell_{N}^{2}-\left(\frac{\ell_{N}+\delta}{2}\right)^{2}\right] .
$$

By Carathéodory's Theorem (see [6]), there exists $I \subset J=\{1, \ldots, k\}$ such that $\operatorname{card}(I) \leq N+2$ and $A$ is a convex combination of $\left\{A_{i}: i \in I\right\}$. We claim that there exist $i, j \in I$ such that

$$
\left|A_{i}-A_{j}\right| \geq\left(\delta+\ell_{N}\right) / 2
$$

Without loss of generality, we assume that

$$
I=\{1, \ldots, N+2\} .
$$

Then take $\left\{d_{i}\right\}_{i=1}^{N+2}$ such that $d_{i} \geq 0$ for all $1 \leq i \leq N+2, \sum_{i=1}^{N+2} d_{i}=1$, and

$$
A=\sum_{i=1}^{N+2} d_{i} A_{i}
$$

It is possible to achieve this, since $A$ is a convex combination of $\left\{A_{i}: 1 \leq i \leq N+2\right\}$. For notational ease, assume as well that $d_{1}=\max \left\{d_{i}: i \in I\right\}$ and $A_{1}=(1,0, \ldots, 0)$. Then since $\sum_{i=1}^{N+2} d_{i}=1$,

$$
d_{1} \geq \frac{1}{N+2} \quad \text { and } \quad \sum_{i=2}^{N+2} d_{i} \leq \frac{N+1}{N+2} .
$$

Set

$$
\gamma=\min \left\{\pi_{1}\left(A_{i}\right): 2 \leq i \leq N+2\right\}
$$

where $\pi_{1}($.$) denotes the first component of a point in \mathbb{R}^{N+1}$. Since $|A| \leq 1 /(N+2)$ (see (2.9)) and $d_{1} \geq 1 /(N+2)$ (see (2.11)), it follows from (2.12) that

$$
\gamma \leq 0
$$

Thus, since

$$
\left|\pi_{1}(A)\right| \geq \sum_{i=1}^{N+2} d_{i} \pi_{1}\left(A_{i}\right) \geq d_{1}+\gamma \sum_{i=2}^{N+2} d_{i}
$$


it follows from (2.9), (2.11) and (2.13) that

$$
\frac{1}{4(N+2)}\left[\ell_{N}^{2}-\left(\frac{\ell_{N}+\delta}{2}\right)^{2}\right] \geq \frac{1}{N+2}+\frac{N+1}{N+2} \gamma
$$

A simple computation yields

$$
\gamma \leq \frac{1}{4}\left[\ell_{N}^{2}-\left(\frac{\ell_{N}+\delta}{2}\right)^{2}\right]-\frac{1}{N+1} .
$$

Take $i_{0} \in\{1, \ldots, N+2\}$ such that $\pi_{1}\left(A_{i_{0}}\right)=\gamma$. Then

$$
\left|A_{1}-A_{i_{0}}\right|^{2}=(1-\gamma)^{2}+\left(1-\gamma^{2}\right)=2-2 \gamma .
$$

On the other hand, from (2.14) and the definition of $\ell_{N}$ (see (1.2)),

$$
2-2 \gamma \geq 2+\frac{2}{N+1}-\frac{1}{2}\left[\ell_{N}^{2}-\left(\frac{\ell_{N}+\delta}{2}\right)^{2}\right]>\ell_{N}^{2}-\left[\ell_{N}^{2}-\left(\frac{\ell_{N}+\delta}{2}\right)^{2}\right] .
$$

Thus

$$
2-2 \gamma>\left(\frac{\delta+\ell_{N}}{2}\right)^{2}
$$

Combining (2.15) and (2.16) yields

$$
\left|A_{1}-A_{i_{0}}\right|>\frac{\delta+\ell_{N}}{2} .
$$

Since $\beta \geq 1$, it follows from the definition of $\varepsilon_{1}$ that

$$
\varepsilon_{1} \leq \frac{1}{16(N+2)}\left[\ell_{N}^{2}-\left(\frac{\ell_{N}+\delta}{2}\right)^{2}\right]=\frac{1}{16(N+2)} \frac{\left(\ell_{N}-\delta\right)\left(3 \ell_{N}+\delta\right)}{4} .
$$

Thus

$$
\varepsilon_{1}<\frac{\ell_{N}-\delta}{2}
$$

which shows that (since $A_{i} \in \Omega_{i}, \operatorname{diam}\left(\Omega_{i}\right) \leq \varepsilon_{1} / 2$ and $\left|A_{1}-A_{i_{0}}\right|>\frac{\delta+\ell_{N}}{2}$ )

$$
\begin{aligned}
|x-y| & \geq\left|A_{1}-A_{i_{0}}\right|-\left|x-A_{1}\right|-\left|y-A_{i_{0}}\right| \\
& >\frac{\delta+\ell_{N}}{2}-\varepsilon_{1} \\
& >\frac{\delta+\ell_{N}}{2}-\frac{\ell_{N}-\delta}{2}=\delta, \quad \forall(x, y) \in \Omega_{1} \times \Omega_{i_{0}} .
\end{aligned}
$$

Therefore, from the construction of $\mathcal{D}_{1}$ and $\mathcal{D}_{i_{0}}$,

$$
\operatorname{meas}(\{(\xi, \eta) \in \mathcal{D} \times \mathcal{D}:|g(\xi)-g(\eta)|>\delta\}) \geq \operatorname{meas}\left(\mathcal{D}_{1} \times \mathcal{D}_{i_{0}}\right) \geq C|\mathcal{D}|^{2} .
$$


Remark 1. Estimate (2.10) can be seen as a perturbation of the result obtained in Lemma 2. A sharper result is proved in Lemma 3.

The following consequence of Lemma 1 is used in the proof of assertion (1.8).

Corollary 2. Let $g \in L^{\infty}\left(\mathbb{S}^{N}, \mathbb{S}^{N}\right), x \in \mathbb{S}^{N}$, and $r \in(0,2)$. Assume that

$$
\left|f_{B(x, r)} g(s) d s\right| \leq \frac{1}{8(N+2)}\left[\ell_{N}^{2}-\left(\frac{\ell_{N}+\delta}{2}\right)^{2}\right],
$$

where $B(x, r)=\left\{y \in \mathbb{S}^{N}:|y-x| \leq r\right\}$.

Then

$$
\operatorname{meas}\left(\left\{(\xi, \eta) \in[B(x, r)]^{2}:|g(\xi)-g(\eta)|>\delta,|\xi-\eta| \geq \tau r\right\}\right) \geq C r^{2 N}
$$

for some positive constants $C=C(\delta, N)$ and $\tau=\tau(\delta, N)$.

Proof. Applying Lemma 1 with $\mathcal{D}=B(x, r)$ and $\beta=1$, one gets

$$
\operatorname{meas}\left(\left\{(\xi, \eta) \in[B(x, r)]^{2}:|g(\xi)-g(\eta)|>\delta\right\}\right) \geq C r^{2 N},
$$

where $C=C(\delta, N)>0$.

It is easy to see that

$$
\operatorname{meas}\left(\left\{(\xi, \eta) \in[B(x, r)]^{2}:|\xi-\eta| \leq \tau r\right\}\right) \leq C_{N} \tau^{N} r^{2 N}, \quad \forall \tau>0 .
$$

Choose $\tau$ such that $C_{N} \tau^{N}=C / 2$. Then it follows from (2.18) and (2.19) that

$$
\operatorname{meas}\left(\left\{(\xi, \eta) \in[B(x, r)]^{2}:|g(\xi)-g(\eta)|>\delta,|\xi-\eta| \geq \tau r\right\}\right) \geq(C / 2) r^{2 N} .
$$

\subsection{Proof of assertion (1.8).}

Step 1. Proof of (1.3) when $g \in \operatorname{Lip}\left(\mathbb{S}^{N}, \mathbb{S}^{N}\right)$.

As in [3], consider the function $u: \mathcal{B} \rightarrow \mathcal{B}$, where $\mathcal{B}=\left\{X \in \mathbb{R}^{N+1}:|X| \leq 1\right\}$, defined by

$$
u(X)=f_{B(x, r)} g(s) d s, \quad \text { when } X \neq 0,
$$

where $x=\frac{X}{|X|}, r=2(1-|X|)$, and

$$
u(0)=f_{\mathbb{S}^{N}} g(s) d s .
$$

We recall that $B(x, r)=\left\{y \in \mathbb{S}^{N}:|y-x| \leq r\right\}$. 
For each $x \in \mathbb{S}^{N}$, let $\rho(x)$ be the length of the largest radial interval coming from $x \in \mathbb{S}^{N}$ on which $|u|>\alpha($ possibly $\rho(x)=1)$, where $\alpha=\frac{1}{8(N+2)}\left[\ell_{N}^{2}-\left(\frac{\ell_{N}+\delta}{2}\right)^{2}\right]$.

In [3], we proved that

$$
|\operatorname{deg} g| \leq \frac{C}{\alpha^{N+1}|\mathcal{B}|} \int_{\substack{\mathbb{S}^{N} \\ \rho(x)<1}} \frac{d x}{\rho(x)^{N}},
$$

where $C=C(N)$ is a constant depending only on $N$.

Take $x \in \mathbb{S}^{N}$ such that $\rho(x)<1$. Then

$$
\left|f_{B(x, 2 \rho(x))} g(s) d s\right|=\alpha .
$$

Thus, by Corollary 2, there exists $\tau=\tau(\delta, N)>0$ such that

$$
\iint_{\substack{[B(x, 2 \rho(x))]^{2} \\|g(\xi)-g(\eta)|>\delta \\|\xi-\eta| \geq \tau \rho(x)}} \frac{1}{|\xi-\eta|^{2 N}} d \xi d \eta \gtrsim 1 .
$$

Here and below, the notation $a \lesssim b$ means that there exists a constant $c$ depending only on $N$ and $\delta$ such that $a \leq c b$. The notation $a \gtrsim b$ means that $b \lesssim a$ and the notation $a \simeq b$ means that $a \lesssim b$ and $b \lesssim a$.

Hence

$$
\int_{\substack{\mathbb{S}^{N} \\ \rho(x)<1}} \frac{1}{\rho(x)^{N}} d x \lesssim \int_{\mathbb{S}^{N}} \frac{1}{\rho(x)^{N}} \underset{\substack{[B(x, 2 \rho(x))]^{2} \\|g(\xi)-g(\eta)|>\delta \\|\xi-\eta| \geq \tau \rho(x)}}{ } \frac{1}{|\xi-\eta|^{2 N}} d \xi d \eta d x
$$

On the other hand, using Fubini's theorem, one gets

$$
\begin{aligned}
\int_{\mathbb{S}^{N}} \frac{1}{\rho(x)^{N}} & \iint_{\substack{[B(x, 2 \rho(x))]^{2} \\
|g(\xi)-g(\eta)|>\delta \\
|\xi-\eta| \geq \tau \rho(x)}} \frac{1}{|\xi-\eta|^{2 N}} d \xi d \eta d x= \\
& \int_{\substack{\mathbb{S}^{N} \\
|g(\xi)-g(\eta)|>\delta}} \int_{\mathbb{S}^{N}} \frac{1}{|\xi-\eta|^{2 N}} \int_{\substack{\mathbb{S}^{N} \\
|x-\xi| \leq 2 \rho(x) \\
|x-\eta| \leq 2 \rho(x) \\
|\xi-\eta| \geq \tau \rho(x)}} \frac{1}{\rho(x)^{N}} d x d \xi d \eta .
\end{aligned}
$$

However, if $|x-\xi| \leq 2 \rho(x),|x-\eta| \leq 2 \rho(x)$ and $|\xi-\eta| \geq \tau \rho(x)$, then $|x-\xi| \leq$ $2|\xi-\eta| / \tau$ and $|\xi-\eta| \leq|x-\xi|+|x-\eta| \leq 4 \rho(x)$. 
Thus

$$
\int_{\substack{\mathbb{S}^{N} \\|x-\xi| \leq 2 \rho(x) \\|x-\eta| \leq 2 \rho(x) \\|\xi-\eta| \geq \tau \rho(x)}} \frac{1}{\rho(x)^{N}} d x \leq \int_{\substack{\mathbb{S}^{N} \\|x-\xi| \leq 2|\xi-\eta| / \tau \\|\xi-\eta| \leq 4 \rho(x)}} \frac{1}{\rho(x)^{N}} d x \lesssim 1
$$

Combining (2.22), (2.23) and (2.24) yields

$$
\int_{\substack{\mathbb{S}^{N} \\ \rho(x)<1}} \frac{1}{\rho(x)^{N}} d x \lesssim \int_{\mathbb{S}^{N}} \int_{\mathbb{S}^{N}} \frac{1}{|g(\xi)-g(\eta)|>\delta} \frac{1}{|\xi-\eta|^{2 N}} d \xi d \eta .
$$

Therefore, (1.8) follows from (2.21) and (2.25).

Step 2. Proof of (1.8) when $g$ is only a continuous function from $\mathbb{S}^{N}$ to $\mathbb{S}^{N}$.

The proof is the same as that of Step 2 in [3, Theorem 1.1].

\section{Construction of a sequence $\left\{g_{k}\right\}_{k \in \mathbb{N}}$ satisfying (1.4) and (1.5).}

We first recall that there exists a regular polyhedron $Q_{N+2}$ which has $N+2$ vertices lying on $\mathbb{S}^{N}$ and each of its edges has length equal to $\ell_{N}=\sqrt{2+2 /(N+1)}$.

Let $A_{1}, A_{2}, \ldots, A_{N+2}$ be the vertices of one of these polyhedrons. Set

$$
F=\bigcup_{i=1}^{N+2} i^{\text {th }} \text {-face } A_{1} \cdots A_{i-1} A_{i+1} \cdots A_{N+2}
$$

and

$$
\begin{gathered}
P: F \rightarrow \mathbb{S}^{N} \\
X \mapsto \frac{X}{|X|},
\end{gathered}
$$

where the $i^{\text {th }}$-face $A_{1} \cdots A_{i-1} A_{i+1} \cdots A_{N+2}$ is, by definition, the set of all the convex combinations of the points $A_{1}, \ldots, A_{i-1}, A_{i+1}, \ldots, A_{N+2}$. Then $P$ is a one-to-one continuous mapping and its inverse mapping is continuous.

Set

$$
D_{i}=-A_{i}, \quad \forall 1 \leq i \leq N+2
$$

and

$$
\Omega_{i}=\left\{P(X): X \in i^{\text {th }} \text {-face } A_{1} \cdots A_{i-1} A_{i+1} \cdots A_{N+2}\right\}
$$

Then

$$
\left|D_{i}-D_{j}\right|=\ell_{N}, \quad \forall 1 \leq i \neq j \leq N+2,
$$


and

$$
\left|x-D_{i}\right| \leq \sqrt{2}, \quad \forall x \in \Omega_{i}, \forall 1 \leq i \leq N+2 .
$$

Moreover,

$$
\left\{\begin{array}{l}
\mathbb{S}^{N}=\bigcup_{i=1}^{N+2} \Omega_{i}, \\
\left|\partial \Omega_{i}\right|=0, \forall 1 \leq i \leq N+2 .
\end{array}\right.
$$

Here $\partial \Omega_{i}$ denotes the boundary of $\Omega_{i}$ corresponding to the usual topology on $\mathbb{S}^{N}$.

For each $x \in \partial \Omega_{i}$, set $\gamma_{x}^{i}=P \circ \hat{\gamma}_{X}^{i}:[0,1] \rightarrow \Omega_{i}$, where $X=P^{-1}(x)$ and $\hat{\gamma}_{X}^{i}$ is the line connecting $X$ and $P^{-1}\left(D_{i}\right)$, i.e., $\hat{\gamma}_{X}^{i}(t)=(1-t) X+t P^{-1}\left(D_{i}\right)$ for all $t \in[0,1]$.

Take

$$
n=2 N+1
$$

For each $k \geq 1$, set $\varepsilon_{k}=\frac{1}{k+1}$ and define $g_{k}$ as follows:

$$
g_{k}\left(\gamma_{x}^{i}(t)\right)= \begin{cases}x & \text { if } 0 \leq t \leq \varepsilon_{k} \\ \gamma_{x}^{i}\left(\left(t-\varepsilon_{k}\right) / \varepsilon_{k}^{n}\right) & \text { if } \varepsilon_{k}<t \leq \varepsilon_{k}+\varepsilon_{k}^{n} \\ D_{i} & \text { if } \varepsilon_{k}+\varepsilon_{k}^{n}<t \leq 1\end{cases}
$$

for all $x \in \partial \Omega_{i}$, for all $1 \leq i \leq N+2$. Then $g_{k}: \mathbb{S}^{N} \rightarrow \mathbb{S}^{N}$ is a continuous mapping and is homotopic to the identity:

$$
\mathcal{I}: \mathbb{S}^{N} \rightarrow \mathbb{S}^{N}
$$

Thus

$$
\operatorname{deg} g_{k}=1
$$

Set (for notational ease)

$$
\left\{\begin{array}{l}
\Omega_{i}^{1}=\left\{\gamma_{x}^{i}(t): x \in \partial \Omega_{i}, \varepsilon_{k}+\varepsilon_{k}^{n}<t \leq 1\right\}, \\
\Omega_{i}^{2}=\left\{\gamma_{x}^{i}(t): x \in \partial \Omega_{i}, \varepsilon_{k}<t \leq \varepsilon_{k}+\varepsilon_{k}^{n}\right\}, \\
\Omega_{i}^{3}=\left\{\gamma_{x}^{i}(t): x \in \partial \Omega_{i}, 0 \leq t \leq \varepsilon_{k}\right\}
\end{array}\right.
$$

Step 1. Estimate of

$$
\iint_{\substack{\Omega_{i} \times \Omega_{i} \\\left|g_{k}(x)-g_{k}(y)\right|>\ell_{N}}} \frac{1}{|x-y|^{2 N}} d x d y,
$$

for $1 \leq i \leq N+2$. 
Since $g_{k}(x)=D_{i}$ for all $x \in \Omega_{i}^{1}$, it follows from (3.1) that

$$
\left|g_{k}(x)-g_{k}(y)\right|<\sqrt{2}, \quad \forall(x, y) \in \Omega_{i}^{1} \times \Omega_{i} .
$$

This implies

$$
\iint_{\substack{\Omega_{i}^{1} \times \Omega_{i} \\\left|g_{k}(x)-g_{k}(y)\right|>\ell_{N}}} \frac{1}{|x-y|^{2 N}} d x d y=0 .
$$

Thus, since $\left\{\Omega_{i}^{l}\right\}_{1 \leq l \leq 3}$ is a partition of $\Omega_{i}$,

$$
\iint_{\substack{\Omega_{i} \times \Omega_{i} \\ g_{k}(x)-g_{k}(y) \mid>\ell_{N}}} \frac{1}{|x-y|^{2 N}} d x d y=\iint_{\substack{\left(\Omega_{i}^{2} \cup \Omega_{i}^{3}\right) \times\left(\Omega_{i}^{2} \cup \Omega_{i}^{3}\right) \\\left|g_{k}(x)-g_{k}(y)\right|>\ell_{N}}} \frac{1}{|x-y|^{2 N}} d x d y .
$$

Since $\gamma_{\xi}^{i}(t)$ is continuous with respect to $\xi$ and $t$ for all $(\xi, t) \in \partial \Omega_{i} \times[0,1]$ and $\operatorname{diam}\left(\left\{\gamma_{\xi}^{i}(t): t \in[0,1]\right\}\right)<\sqrt{2}<\ell_{N}$, there exists a constant $\delta(N)$ such that

(3.5) if $|\xi-\eta|<\delta(N)$, then $\left|\gamma_{\xi}^{i}(t)-\gamma_{\eta}^{i}(s)\right|<\ell_{N}, \quad \forall(\xi, \eta) \in \partial \Omega_{i}, \forall s, t \in[0,1]$.

Define the mapping $G_{i}$ as

$$
\begin{aligned}
G_{i}: \Omega_{i} \backslash\left\{D_{i}\right\} \longrightarrow \partial \Omega_{i} \\
x \longmapsto G_{i}(x),
\end{aligned}
$$

where $G_{i}(x) \in \partial \Omega_{i}$ is the point such that $P^{-1}(x)$ is a convex combination of $P^{-1}\left(G_{i}(x)\right)$ and $P^{-1}\left(D_{i}\right)$. Then, since $G_{i}$ is a continuous map on $\Omega_{i} \backslash\left\{D_{i}\right\}$, there exists a constant $C$ such that

(3.6) if $|x-y|<C$, then $\left|G_{i}(x)-G_{i}(y)\right|<\delta(N), \quad \forall(x, y) \in\left(\Omega_{i}^{2} \cup \Omega_{i}^{3}\right) \times\left(\Omega_{i}^{2} \cup \Omega_{i}^{3}\right)$.

Henceforth in this proof, $C$ denotes a positive constant depending only on $N$.

On the other hand, for each $(x, y) \in\left(\Omega_{i}^{2} \cup \Omega_{i}^{3}\right) \times\left(\Omega_{i}^{2} \cup \Omega_{i}^{3}\right)$, it follows from the definition of $g_{k}$ that there exist some $s, t \in[0,1]$ such that

$$
g_{k}(x)=\gamma_{G_{i}(x)}(t) \quad \text { and } \quad g_{k}(y)=\gamma_{G_{i}(y)}(s) .
$$

Hence, combining (3.5)-(3.7) yields that $\left|g_{k}(x)-g_{k}(y)\right| \leq \ell_{N}$ whenever $|x-y| \leq C$ and $x, y \in \Omega_{i}^{2} \cup \Omega_{i}^{3}$.

Thus, since $\left|\Omega_{i}^{2} \cup \Omega_{i}^{3}\right| \lesssim \varepsilon_{k}$, one deduces that

$$
\iint_{\substack{\left(\Omega_{i}^{2} \cup \Omega_{i}^{3}\right) \times\left(\Omega_{i}^{2} \cup \Omega_{i}^{3}\right) \\\left|g_{k}(x)-g_{k}(y)\right|>\ell_{N}}} \frac{1}{|x-y|^{2 N}} d x d y=\iint_{\substack{\left(\Omega_{i}^{2} \cup \Omega_{i}^{3}\right) \times\left(\Omega_{i}^{2} \cup \Omega_{i}^{3}\right) \\\left|g_{k}(x)-g_{k}(y)\right|>\ell_{N} \\|x-y| \geq C}} \frac{1}{|x-y|^{2 N}} d x d y \lesssim \varepsilon_{k}^{2} .
$$


Combining (3.4) and (3.8) yields

$$
\iint_{\substack{\Omega_{i} \times \Omega_{i} \\\left|g_{k}(x)-g_{k}(y)\right|>\ell_{N}}} \frac{1}{|x-y|^{2 N}} d x d y \lesssim \varepsilon_{k}^{2} .
$$

Step 2. Estimate of

$$
\iint_{\substack{\Omega_{i} \times \Omega_{j} \\\left|g_{k}(x)-g_{k}(y)\right|>\ell_{N}}} \frac{1}{|x-y|^{2 N}} d x d y,
$$

for $1 \leq i \neq j \leq N+2$.

Since $\left|D_{i}-D_{j}\right|=\ell_{N}, g_{k}(x)=D_{i}$ and $g_{k}(y)=D_{j}$ for all $(x, y) \in \Omega_{i}^{1} \times \Omega_{j}^{1}$,

$$
\iint_{\substack{\Omega_{i}^{1} \times \Omega_{j}^{1} \\\left|g_{k}(x)-g_{k}(y)\right|>\ell_{N}}} \frac{1}{|x-y|^{2 N}} d x d y=0 .
$$

On the other hand, since $\left|\Omega_{i}^{2}\right| \lesssim \varepsilon_{k}^{n}$ and $|x-y| \gtrsim \varepsilon_{k}$ for all $(x, y) \in \Omega_{i}^{2} \times \Omega_{j}$, we infer that

$$
\iint_{\substack{\Omega_{i}^{2} \times \Omega_{j} \\\left|g_{k}(x)-g_{k}(y)\right|>\ell_{N}}} \frac{1}{|x-y|^{2 N}} d x d y \lesssim \varepsilon_{k}^{n-2 N}=\varepsilon_{k} .
$$

Set $\Gamma_{i j}=\partial \Omega_{i} \cap \partial \Omega_{j}$. Then

$$
\left|z-D_{i}\right|<\sqrt{2}, \quad \forall z \in \Gamma_{i j} .
$$

Hence $\left|z-D_{i}\right|<\ell_{N}$ for all $z \in \Omega_{j}$ such that $\operatorname{dist}\left(z, \Gamma_{i j}\right) \leq C$.

This implies (since $\left|\Omega_{i}^{3}\right| \lesssim \varepsilon_{k}$ )

$$
\iint_{\substack{\Omega_{i}^{1} \times \Omega_{j}^{3} \\\left|g_{k}(x)-g_{k}(y)\right|>\ell_{N}}} \frac{1}{|x-y|^{2 N}} d x d y \lesssim \varepsilon_{k} .
$$

Combining (3.10)-(3.12) yields

$$
\iint_{\substack{\Omega_{i} \times \Omega_{j} \\\left|g_{k}(x)-g_{k}(y)\right|>\ell_{N}}} \frac{1}{|x-y|^{2 N}} d x d y \lesssim \varepsilon_{k} .
$$

Thus it follows from (3.9) and (3.13) that

$$
\iint_{\substack{\mathbb{S}^{N} \times \mathbb{S}^{N} \\\left|g_{k}(x)-g_{k}(y)\right|>\ell_{N}}} \frac{1}{|x-y|^{2 N}} d x d y \lesssim \varepsilon_{k} .
$$


Therefore, from (3.3) and (3.14), the sequence $\left\{g_{k}\right\}_{k \in N}$ satisfies (1.4) and (1.5).

Remark 2. Given any $\varphi \in C(\mathbb{R} \backslash\{0\})$, the estimate

$$
|\operatorname{deg} g| \leq C \int_{|g(x)-g(y)|>\ell_{N}} \int_{\mathbb{S}^{N}} \varphi(|x-y|) d x d y, \quad \forall g \in C\left(\mathbb{S}^{N}, \mathbb{S}^{N}\right),
$$

where $C$ is a positive constant independent of $g$, fails. To see this, one can use the same construction of a sequence $\left\{g_{k}\right\}_{k \in N}$ as above with an appropriate choice of $n$.

Remark 3. Theorem 1 shows that the condition $\delta<\sqrt{2}$ is optimal, if we want a condition independent of $N$ (see [3]).

Remark 4. In [4] H. Brezis raised the following interesting question related to the behavior of the constant $C=C(\delta, N)$ as $\delta$ goes to 0 , in the estimate (1.8). Does there exist a positive constant $C=C(N)$ such that

$$
|\operatorname{deg} g| \leq C \int_{|g(x)-g(y)|>\delta} \int_{\mathbb{S}^{N}} \frac{\delta^{N}}{|x-y|^{2 N}} d x d y, \quad \forall g \in C\left(\mathbb{S}^{N}, \mathbb{S}^{N}\right), \forall 0<\delta<1 ?
$$

\section{Proof of assertion (1.3)}

In this section, we prove that the constant $C=C(\delta, N)$ in (1.8) can be chosen independently of $\delta$. This completes the proof of Theorem 1. We first prove some preliminary results.

4.1 Preliminaries. We begin this section with the following lemma, which deals with a nice geometric property of $\mathbb{S}^{N}$. The idea of the proof has already appeared in the proof of Lemma 1 . In this section, $O$ denotes the point $(0, \ldots, 0) \in \mathbb{R}^{N+1}$ and conv (.) denotes the convex hull of a subset of $\mathbb{R}^{N+1}$.

Lemma 2. Let $A_{i} \in \mathbb{S}^{N}, 1 \leq i \leq N+2$, be such that $O$ is a convex combination of $\left\{A_{i}\right\}_{i=1}^{N+2}$. Then there exist $1 \leq i, j \leq N+2$ such that

$$
\left|A_{i}-A_{j}\right| \geq \ell_{N} .
$$

Proof. Since $O$ is a convex combination of $A_{i}$, there exist $\left\{d_{i}\right\}_{i=1}^{N+2}$ such that $d_{i} \geq 0$, for all $1 \leq i \leq N+2, \sum_{i=1}^{N+2} d_{i}=1$, and

$$
O=\sum_{i=1}^{N+2} d_{i} A_{i}
$$


Without loss of generality, we assume that $d_{1}=\max \left\{d_{i}: 1 \leq i \leq N+2\right\}$ and $A_{1}=(1,0, \ldots, 0)$. Then, since $\sum_{i=1}^{N+2} d_{i}=1$,

$$
d_{1} \geq \frac{1}{N+2} \quad \text { and } \quad \sum_{i=2}^{N+2} d_{i} \leq \frac{N+1}{N+2} .
$$

Set

$$
\gamma=\min \left\{\pi_{1}\left(A_{i}\right): 2 \leq i \leq N+2\right\},
$$

where as before $\pi_{1}($.$) denotes the first component of a point in \mathbb{R}^{N+1}$. Then it is easy to see that

$$
\gamma \leq 0 .
$$

Thus, since

$$
0=\sum_{i=1}^{N+2} d_{i} \pi_{1}\left(A_{i}\right) \geq d_{1}+\gamma \sum_{i=2}^{N+2} d_{i}
$$

it follows from (4.1), (4.2) and (4.3) that

$$
0 \geq \frac{1}{N+2}+\frac{N+1}{N+2} \gamma .
$$

A simple computation yields

$$
\gamma \leq-\frac{1}{N+1}
$$

Take $i_{0} \in\{1, \ldots, N+2\}$ such that $\pi_{1}\left(A_{i_{0}}\right)=\gamma$. Then

$$
\left|A_{1}-A_{i_{0}}\right|^{2}=(1-\gamma)^{2}+\left(1-\gamma^{2}\right)=2-2 \gamma \geq 2+\frac{2}{N+1} .
$$

Therefore, $\left|A_{1}-A_{i_{0}}\right| \geq \ell_{N}$

The following result is an important improvement of Lemma 2, which is used in the proof of Lemma 5.

Lemma 3. Let $A_{i} \in \mathbb{S}^{N}, 1 \leq i \leq N+2$. Assume that there exists a convex combination $A$ of $\left\{A_{i}\right\}_{i=1}^{N+2}$ such that $|A| \leq \frac{1}{N+1}$. Then there exist $1 \leq i, j \leq N+2$, such that

$$
\left|A_{i}-A_{j}\right| \geq \ell_{N}
$$

Proof. Set

$$
\mathbf{K}=\operatorname{conv}\left(\left\{A_{i}: 1 \leq i \leq N+2\right\}\right) .
$$

Then $\mathbf{K}$ is a convex, closed, and non-empty subset of $\mathbb{R}^{N+1}$. 
If $O \in \mathbf{K}$, then applying Lemma 2, one has

$$
\left|A_{i}-A_{j}\right| \geq \ell_{N}
$$

for some $1 \leq i, j \leq N+2$.

We now suppose that $O \notin \mathbf{K}$. Let $H$ be the projection of $O$ onto $\mathbf{K}$, i.e.,

$$
\frac{1}{2}|H|^{2}=\frac{1}{2} \min _{X \in \mathbf{K}}|X|^{2} .
$$

Let $\mathbf{P}$ be the hyperplane containing $H$ such that $O H$ is orthogonal to $\mathbf{P}$. Set

$$
\mathbb{S}=\mathbf{P} \cap \mathbb{S}^{N}
$$

Then $H$ is the center of $\mathbb{S}$; moreover, $H$ is also a convex combination of $\left\{A_{i}: 1 \leq i \leq N+2, A_{i} \in \mathbf{P}\right\}$. Applying Lemma 2, one gets

$$
\left|A_{i}-A_{j}\right|^{2} \geq\left(1-|H|^{2}\right)(2+2 / N) \text {. }
$$

for some $1 \leq i, j \leq N+2$. On the other hand,

$$
|H| \leq|A| \leq 1 /(N+1)
$$

Thus

$$
\left|A_{i}-A_{j}\right|^{2} \geq(2+2 /(N+1))
$$

which shows that

$$
\left|A_{i}-A_{j}\right| \geq \ell_{N}
$$

for some $1 \leq i, j \leq N+2$.

Remark 5. It is easy to see that the condition $|A| \leq \frac{1}{N+1}$ in Lemma 3 is optimal.

The following result is a consequence of Lemma 3 and Carathéodory's Theorem (see [6]).

Corollary 3. Let I be a nonempty set and $A_{i} \in \mathbb{S}^{N}$, for all $i \in I$. Assume that there exists $A \in \operatorname{conv}\left(\left\{A_{i}: i \in I\right\}\right)$, such that $|A| \leq \frac{1}{N+1}$. Then

$$
\left|A_{i}-A_{j}\right| \geq \ell_{N}
$$

for some $i, j \in I$. 
Proof. Since $A \in \operatorname{conv}\left(\left\{A_{i} ; i \in I\right\}\right)$, by Carathéodory's Theorem, there exist $\left\{i_{m}\right\}_{m=1}^{N+2} \subset I$ such that $A$ is a convex combination of $\left\{A_{i_{m}}\right\}_{m=1}^{N+2}$. Thus, applying Lemma 2, one has

$$
\left|A_{i_{l}}-A_{i_{m}}\right| \geq \ell_{N}
$$

for some $1 \leq l, m \leq N+2$.

Another geometric property of $\mathbb{S}^{N}$ is given in the following lemma

Lemma 4. Let $A_{i} \in \mathbb{S}^{N}, 1 \leq i \leq N+2$. Then there exist $1 \leq i \neq j \leq N+2$ such that

$$
\left|A_{i}-A_{j}\right| \leq \ell_{N}
$$

Proof. We prove this by contradiction. Suppose that

$$
\left|A_{i}-A_{j}\right|>\ell_{N}, \quad \forall 1 \leq i \neq j \leq N+2 .
$$

Then, from (1.2),

$$
\left|A_{i}\right|^{2}-2 A_{i} \cdot A_{j}+\left|A_{j}\right|^{2}>2+\frac{2}{N+1}, \quad \forall 1 \leq i \neq j \leq N+2,
$$

where $A_{i} \cdot A_{j}$ denotes the scalar product of $A_{i}$ and $A_{j}$. Consequently,

$$
A_{i} \cdot A_{j}<-\frac{1}{N+1}, \quad \forall 1 \leq i \neq j \leq N+2 .
$$

This implies, for all $1 \leq i \leq N+2$,

$$
\sum_{j=1}^{N+2} A_{i} \cdot A_{j}=\sum_{\substack{j=1 \\ j \neq i}}^{N+2} A_{i} \cdot A_{j}+\left|A_{i}\right|^{2}<-\frac{N+1}{N+1}+1=0 .
$$

Thus

$$
\left|\sum_{i=1}^{N+2} A_{i}\right|^{2}=\sum_{i=1}^{N+2} \sum_{j=1}^{N+2} A_{i} \cdot A_{j}<0 .
$$

We have a contradiction.

We now introduce some positive constants depending only on $N$ which are used later.

Define

$$
c_{N}=1-\sup _{\substack{t \in\left[\frac{1}{N+1}, 1\right] \\ s \in\left[-1, \frac{1}{2(N+1)}\right]}} \frac{\sqrt{1-s^{2}}}{\sqrt{1-s^{2}+(t-s)^{2}}}>0 .
$$


Next fix $C(N)$ and $b_{N} \in \mathbb{N}$, two positive constants depending only on $N$ such that

$$
|B(x, r)| \geq C(N) r^{N}, \quad \forall x \in \mathbb{S}^{N}, \forall 0<r<1,
$$

and

$$
\left|\mathbb{S}^{N}\right|<C(N) b_{N}\left[\frac{c_{N}}{2(N+1)}\right]^{N},
$$

where $c_{N}$ is defined by (4.4).

The next lemma has a consequence (Corollary 4) which is useful in the proof of Lemma 6.

Lemma 5. Let $k \in \mathbb{N}_{+}$and $A_{i} \in \mathbb{S}^{N}$ for all $1 \leq i \leq k$. Assume that $k \geq$ $16 b_{N}(N+1)$ and

$$
\left|\sum_{i=1}^{k} \frac{1}{k} A_{i}\right| \leq \frac{1}{16(N+1)} .
$$

Then there exists $1 \leq i_{0} \leq k$ such that

$$
\operatorname{card}\left(\left\{1 \leq i \leq k:\left|A_{i}-A_{i_{0}}\right| \geq \ell_{N}\right\}\right) \geq \frac{k}{4 b_{N}(N+1)} .
$$

Proof. Set $I=\{i \in \mathbb{N}: 1 \leq i \leq k\}$ and

$$
U_{n}=\left\{i \in I:\left|A_{i}-A_{n}\right| \geq \ell_{N}\right\}, \quad \forall n \in I .
$$

We argue by contradiction. Suppose that

$$
\operatorname{card}\left(U_{i}\right)<\frac{k}{4 b_{N}(N+1)}, \quad \forall i \in I .
$$

Take $i_{n} \in I$ and $V_{n} \subset I, 1 \leq n \leq b_{N}$, such that $i_{1}=1, V_{1}=I \backslash U_{i_{1}}, i_{n+1} \in I \backslash V_{n}$ satisfies

$$
\begin{aligned}
\operatorname{dist}\left(O, \operatorname{conv}\left(\left\{A_{i_{m}}: 1 \leq m\right.\right.\right. & \leq n+1\}))= \\
& \min _{j \in I \backslash V_{n}} \operatorname{dist}\left(O, \operatorname{conv}\left(\left\{A_{i_{m}}: 1 \leq m \leq n\right\} \cup\left\{A_{j}\right\}\right)\right),
\end{aligned}
$$

and

$$
V_{n+1}=V_{n} \cup U_{i_{n+1}},
$$

for all $1 \leq n<b_{N}$ (since $k \geq 16 b_{N}(N+1)$, it follows from (4.7) that $I \backslash V_{n} \neq \emptyset$, for all $\left.1 \leq n \leq b_{N}\right)$.

Set

$$
\delta_{n}=\operatorname{dist}\left(O, \operatorname{conv}\left(\left\{A_{i_{m}}: 1 \leq m \leq n\right\}\right)\right) .
$$


Then, since $\left|A_{i_{m}}-A_{i_{n}}\right|<\ell_{N}$ for all $1 \leq m, n \leq b_{N}$, it follows by Corollary 3 that

$$
\delta_{n} \geq \frac{1}{N+1}, \quad \forall 1 \leq n \leq b_{N} .
$$

We claim that $\left|A_{i_{m}}-A_{i_{n}}\right| \geq c_{N} /(N+1)$ for all $1 \leq m<n \leq b_{N}$, where $c_{N}$ is defined by (4.4).

In fact, suppose that

$$
\left|A_{i_{m}}-A_{i_{n}}\right|<c_{N} /(N+1) \text { for some } 1 \leq m<n \leq b_{N} .
$$

Let $H_{n}$ be the projection of $O$ onto conv $\left(\left\{A_{i_{m}}: 1 \leq m \leq n-1\right\}\right)$. Without loss of generality, we assume that $H_{n}=\left(h_{n}, 0, \ldots, 0\right)$ and $h_{n} \geq 0$. Then

$$
H_{n} \cdot\left(A_{i_{l}}-H_{n}\right) \geq 0, \quad \forall 1 \leq l \leq n-1 .
$$

Thus, from (4.8) and (4.9), this implies

$$
\delta_{n}>h_{n}-c_{N} /(N+1) \geq h_{n}-c_{N} h_{n} .
$$

Therefore,

$$
\pi_{1}\left(A_{i}\right) \geq \frac{1}{2(N+1)}, \quad \forall i \in I \backslash V_{n-1} .
$$

To see this, suppose that $\pi_{1}\left(A_{i}\right)<\frac{1}{2(N+1)}$, for some $i \in I \backslash V_{n-1}$. Then

$$
\operatorname{dist}\left(O, \operatorname{conv}\left(\left\{A_{i_{m}}: 1 \leq m \leq n-1\right\} \cup\left\{A_{i}\right\}\right)\right) \leq \operatorname{dist}\left(O, H_{n} A_{i}\right) .
$$

Here $H_{n} A_{i}$ denotes the segment whose end points are $H_{n}$ and $A_{i}$, i.e.,

$$
H_{n} A_{i}=\left\{t H_{n}+(1-t) A_{i}: 0 \leq t \leq 1\right\} .
$$

Thus, from (4.4) and (4.8),

$$
\operatorname{dist}\left(O, H_{n} A_{i}\right) \leq h_{n}\left(1-c_{N}\right) .
$$

Combining (4.10), (4.11), and (4.12) yields

$$
\operatorname{dist}\left(O, \operatorname{conv}\left(\left\{A_{i_{m}}: 1 \leq m \leq n-1\right\} \cup A_{i}\right)\right)<\delta_{n} .
$$

This contradicts the definition of $\delta_{n}$. Thus

$$
\pi_{1}\left(A_{i}\right) \geq \frac{1}{2(N+1)}, \quad \forall i \in I \backslash V_{n-1} .
$$

Hence one deduces from (4.7) and (4.13) that

$$
\left|\sum_{i=1}^{k} \frac{1}{k} A_{i}\right| \geq\left|\sum_{i \in I \backslash V_{n-1}} \frac{1}{k} A_{i}\right|-\left|\sum_{i \in V_{n-1}} \frac{1}{k} A_{i}\right| \geq \frac{k-\operatorname{card}\left(V_{n-1}\right)}{2(N+1)}-\frac{b_{N}}{k} \frac{k}{4 b_{N}(N+1)} .
$$


On the other hand, since $k \geq 16 b_{N}(N+1)$ and $n \leq b_{N}$, it follows from (4.7) that

$$
k-\operatorname{card}\left(V_{n-1}\right) \geq k-b_{N} \frac{k}{4 b_{N}(N+1)}=k-\frac{k}{4(N+1)} \geq \frac{2 k}{3} .
$$

Thus, from (4.14), one has

$$
\left|\sum_{i=1}^{k} \frac{1}{k} A_{i}\right| \geq \frac{1}{3(N+1)}-\frac{1}{4(N+1)}=\frac{1}{12(N+1)} .
$$

This contradicts (4.6). Hence

$$
\left|A_{i_{m}}-A_{i_{n}}\right| \geq c_{N} /(N+1), \quad \forall 1 \leq m \neq n \leq b_{N} .
$$

Thus we obtain a family $\left\{B\left(A_{i_{m}}, \frac{c_{N}}{2(N+1)}\right)\right\}_{i=1}^{b_{N}}$ such that

$$
B\left(A_{i_{m}}, \frac{c_{N}}{2(N+1)}\right) \cap B\left(A_{i_{n}}, \frac{c_{N}}{2(N+1)}\right)=\emptyset, \quad \forall 1 \leq m \neq n \leq b_{N} .
$$

Therefore,

$$
\left|\mathbb{S}^{N}\right| \geq C(N) b_{N}\left[\frac{c_{N}}{2(N+1)}\right]^{N}
$$

This contradicts the choice of $b_{N}$.

Remark 6. Applying Corollary 3, we deduce from (4.6) that there exist $1 \leq i, j \leq k$ such that $\left|A_{i}-A_{j}\right| \geq \ell_{N}$. However, under the condition (4.6), Lemma 5 gives more information: There exists $1 \leq i_{0} \leq k$ such that $\operatorname{card}\left(\left\{1 \leq i \leq k:\left|A_{i}-A_{i_{0}}\right| \geq \ell_{N}\right\}\right) \geq C_{N} k$ whenever $k$ is sufficiently big, where $C_{N}$ is a positive constant depending only on $N$.

Corollary 4. Let $k \in \mathbb{N}_{+}$and $A_{i} \in \mathbb{S}^{N}$, for $1 \leq i \leq k$. Assume that $k \geq$ $100 b_{N}(N+1)$ and

$$
\left|\sum_{i=1}^{k} \frac{1}{k} A_{i}\right| \leq \frac{1}{64(N+1)}
$$

Then

$$
\operatorname{card}\left(\left\{(i, j): 1 \leq i, j \leq k,\left|A_{i}-A_{j}\right| \geq \ell_{N}\right\}\right) \geq \frac{k^{2}}{1000 b_{N}^{2}(N+1)^{2}} .
$$

Proof. Set

$$
I=\{i \in \mathbb{N}: 1 \leq i \leq k\}
$$

and

$$
U_{n}=\left\{i \in I:\left|A_{i}-A_{n}\right| \geq \ell_{N}\right\}, \quad \forall n \in I .
$$


By Lemma 5, there exists $i_{1} \in I$ such that

$$
\operatorname{card}\left(U_{i_{1}}\right) \geq \frac{k}{4 b_{N}(N+1)}
$$

Suppose that there exist $i_{m}, 1 \leq m \leq n\left(n<\frac{k}{50(N+1)}\right)$, such that $i_{m} \neq i_{l}$ for $m \neq l$ and $\operatorname{card}\left(U_{i_{m}}\right) \geq \frac{1}{8 b_{N}(N+1)}$. Set

$$
I_{n}=\left\{i_{m}: 1 \leq m \leq n\right\} .
$$

One has

$$
\sum_{i \in I \backslash I_{n}} \frac{1}{k-n} A_{i}=\frac{k}{k-n} \sum_{i \in I} \frac{1}{k} A_{i}-\sum_{i \in I_{n}} \frac{1}{k-n} A_{i}
$$

Since $n<\frac{k}{50(N+1)}$, it follows that

$$
\left|\frac{k}{k-n} \sum_{i \in I} \frac{1}{k} A_{i}\right| \leq \frac{2}{64(N+1)}=\frac{1}{32(N+1)}
$$

(by (4.16)) and

$$
\left|\sum_{i \in I_{n}} \frac{1}{k-n} A_{i}\right| \leq \frac{n}{k-n} \leq \frac{1}{32(N+1)}
$$

Combining (4.17), (4.18), and (4.19) yields

$$
\left|\sum_{i \in I \backslash I_{n}} \frac{1}{k-n} A_{i}\right| \leq \frac{1}{16(N+1)} .
$$

Thus, since $n<\frac{k}{50(N+1)}$ and $k \geq 100 b_{N}(N+1)$, it follows that $k-n \geq k / 2$ and $k-n \geq 16 b_{N}(N+1)$. Hence, by Lemma 5, there exists $i_{n+1} \in I \backslash I_{n}$ such that

$$
\operatorname{card}\left(U_{i_{n+1}}\right) \geq \frac{k}{8 b_{N}(N+1)}
$$

Therefore, there exist $i_{m}, 1 \leq m \leq \frac{k}{50(N+1)}$, such that $i_{m} \neq i_{l}$ for $l \neq m$ and

$$
\operatorname{card}\left(U_{i_{m}}\right) \geq \frac{k}{8 b_{N}(N+1)}, \quad \forall 1 \leq m \leq \frac{k}{50(N+1)} .
$$

Consequently,

$$
\operatorname{card}\left(\left\{(i, j): 1 \leq i, j \leq k,\left|A_{i}-A_{j}\right| \geq \ell_{N}\right\}\right) \geq \frac{k^{2}}{1000 b_{N}^{2}(N+1)^{2}}
$$

The following result is a continuous version of Corollary 4 . 
Lemma 6. Let $g \in L^{\infty}\left(\mathbb{S}^{N}, \mathbb{S}^{N}\right)$, D be a measurable subset of $\mathbb{S}^{N}$ with $|\mathcal{D}|>0$, and $\lambda: \mathcal{D} \rightarrow[0,+\infty)$ be a measurable function. Assume that there exists a constant $\beta \geq 1$ such that $1 / \beta \leq \lambda(s) \leq \beta$ for every $s \in \mathcal{D}$ and

$$
\left|f_{\mathcal{D}} \lambda(s) g(s) d s\right| \leq \frac{1}{128 \beta(N+1)} .
$$

Then

$$
\operatorname{meas}(\{(\xi, \eta) \in \mathcal{D} \times \mathcal{D}:|g(\xi)-g(\eta)|>\delta\}) \geq C|\mathcal{D}|^{2}
$$

for some $C=C(\beta, N)$.

Proof. Let $\Omega_{1}, \ldots, \Omega_{k_{1}}$ be the measurable subsets of $\mathbb{S}^{N}$ such that $\mathbb{S}^{N}=$ $\bigcup_{i=1}^{k_{1}} \Omega_{i}, \Omega_{i} \cap \Omega_{j}=\emptyset$, for each $1 \leq i \neq j \leq k_{1}$, and

$$
\operatorname{diam}\left(\Omega_{i}\right)<\min \left\{\frac{1}{128 \beta^{2}(N+1)}, \frac{\ell_{N}-\delta}{3}\right\}
$$

for every $1 \leq i \leq k_{1}$. Set

$$
\mathcal{D}_{i}=g^{-1}\left(\Omega_{i}\right) \cap \mathcal{D}, \quad \forall 1 \leq i \leq k_{1} .
$$

We assume as well that

$$
\left|\mathcal{D}_{i}\right|>0, \quad \forall 1 \leq i \leq k,
$$

for some $k \leq k_{1}$. Take $A_{i} \in \mathcal{D}_{i}$. Then

$$
\left|\sum_{i=1}^{k} \int_{\mathcal{D}_{i}} \lambda(s) d s A_{i}\right| \leq\left|\sum_{i=1}^{k} \int_{\mathcal{D}_{i}} \lambda(s) g(s) d s\right|+\left|\sum_{i=1}^{k} \int_{\mathcal{D}_{i}} \lambda(s)\left(g(s)-A_{i}\right) d s\right|,
$$

which shows ( since $1 / \beta \leq \lambda(s) \leq \beta$ for all $s \in \mathcal{D}$ ) that

$$
\left|\sum_{i=1}^{k} c_{i} A_{i}\right| \leq \beta\left|f_{\mathcal{D}} \lambda(s) g(s) d s\right|+\beta^{2} \max _{i} \operatorname{diam}\left(\Omega_{i}\right),
$$

where

$$
c_{i}=\left(\int_{\mathcal{D}_{i}} \lambda(s) d s\right) /\left(\sum_{i=1}^{k} \int_{\mathcal{D}_{i}} \lambda(s) d s\right) .
$$

Consequently,

$$
\left|\sum_{i=1}^{k} c_{i} A_{i}\right|<\frac{1}{64(N+1)}
$$

Without loss of generality, suppose that $c_{i}, 1 \leq i \leq k$, is a rational number (otherwise, we may approximate $c_{i}, 1 \leq i \leq k$, by rational numbers). Suppose that $c_{i}=p_{i} / q, p_{i} \in \mathbb{N}_{+}$and $q \in \mathbb{N}_{+}(q \gg N)$, for all $1 \leq i \leq k$. We as well assume that 
$p_{i}=1$ for all $1 \leq i \leq k$ (if not, take $\Omega_{i}^{j}, 1 \leq j \leq p_{i}$, such that $\bigcup_{j=1}^{p_{i}} \Omega_{i}^{j}=\Omega_{i}$ and $\int_{\mathcal{D}_{i}^{j}} \lambda(s) d s$ approximates $1 / q$, where $\mathcal{D}_{i}^{j}=g^{-1}\left(\Omega_{i}^{j}\right)$, and $A_{i}^{j}=A_{i}$, for $\left.1 \leq j \leq p_{i}\right)$. Thus we now suppose that $c_{i}=1 / k$ for all $1 \leq i \leq k$, and $k \geq 100 b_{N}(N+1)$. Then

$$
\left|\sum_{i=1}^{k} \frac{1}{k} A_{i}\right|<\frac{1}{64(N+1)}
$$

Applying Corollary 4, one has

$$
\operatorname{card}\left(\left\{(i, j): 1 \leq i, j \leq k,\left|A_{i}-A_{j}\right| \geq \ell_{N}\right\}\right) \geq C_{N} k^{2} .
$$

On the other hand, since $c_{i}=1 / k$ and $1 / \beta \leq \lambda(s) \leq \beta$ for $s \in \mathcal{D}$, one sees from the definition of $c_{i}$ that

$$
\frac{1}{k}=c_{i} \leq \frac{\beta^{2}\left|\Omega_{i}\right|}{|\mathcal{D}|},
$$

which shows

$$
\left|\Omega_{i}\right| \geq \frac{1}{\beta^{2} k}|\mathcal{D}| .
$$

Since $\Omega_{i} \cap \Omega_{j}=\emptyset$ for $i \neq j$, combining (4.20) and (4.21) yields

$$
\operatorname{meas}\left(\left\{(x, y): x \in \Omega_{i}, y \in \Omega_{j},\left|A_{i}-A_{j}\right| \geq \ell_{N}\right\}\right) \geq C_{N} k^{2} \frac{1}{\beta^{4} k^{2}}|\mathcal{D}|^{2}=C_{N, \beta}|\mathcal{D}|^{2} .
$$

Therefore, since $\operatorname{diam}\left(\Omega_{i}\right) \leq\left(\ell_{N}-\delta\right) / 3$, it follows that

$$
\operatorname{meas}(\{(\xi, \eta) \in \mathcal{D} \times \mathcal{D}:|g(\xi)-g(\eta)|>\delta\}) \geq C_{N, \beta}|\mathcal{D}|^{2} .
$$

\subsection{Proof of assertion (1.3).}

Step 1. Proof of assertion (1.3) when $g \in \operatorname{Lip}\left(\mathbb{S}^{N}, \mathbb{S}^{N}\right)$.

As in [3], consider the function $u: \mathcal{B} \rightarrow \mathcal{B}$, where $\mathcal{B}=\left\{X \in \mathbb{R}^{N+1}:|X| \leq 1\right\}$, defined by

$$
u(X)=f_{B(x, r)} g(s) d s, \quad \text { when } X \neq 0,
$$

where $x=X /|X|, r=2(1-|X|)$, and

$$
u(0)=f_{\mathbb{S}^{N}} g(s) d s .
$$

For each $x \in \mathbb{S}^{N}$, let $\rho(x)$ be the length of the largest radial interval coming from $x \in \mathbb{S}^{N}$ on which $|u|>\frac{1}{128(N+1)}$ (possibly $\rho(x)=1$ ). 
Thus, as in [3], one has

$$
|\operatorname{deg} g| \leq C \int_{\substack{\mathbb{S}^{N} \\ \rho(x)<1}} \frac{d x}{\rho(x)^{N}}
$$

Hereafter $C$ denotes a constant depending only on $N$ in this proof. Take $x \in \mathbb{S}^{N}$ such that $\rho(x)<1$. Then

$$
\left|f_{B(x, 2 \rho(x))} g(s) d s\right|=\frac{1}{128(N+1)} .
$$

Applying Lemma 6, one has

$$
\text { meas }\left\{(\xi, \eta) \in[B(x, 2 \rho(x))]^{2}:|g(\xi)-g(\eta)| \geq \delta\right\} \geq C \rho(x)^{2 N} .
$$

Hence there exists a constant $\tau=\tau(N)$, depending only on $N$, such that

$$
\text { meas }\left\{(\xi, \eta) \in[B(x, 2 \rho(x))]^{2}:|g(\xi)-g(\eta)| \geq \delta,|\xi-\tau| \geq \tau \rho(x)\right\} \geq C \rho(x)^{2 N} .
$$

This implies

$$
\iint_{\substack{[B(x, 2 \rho(x))]^{2} \\|g(\xi)-g(\eta)|>\delta \\|\xi-\eta| \geq \tau \rho(x)}} \frac{1}{|\xi-\eta|^{2 N}} d \xi d \eta \geq C .
$$

Combining (4.23) and (4.24) yields

$$
|\operatorname{deg} g| \leq C \int_{\mathbb{S}^{N}} \frac{1}{\rho(x)^{N}} \underset{\substack{[B(x, 2 \rho(x))]^{2} \\|g(\xi)-g(\eta)|>\delta \\|\xi-\eta| \geq \tau \rho(x)}}{ } \frac{1}{|\xi-\eta|^{2 N}} d \xi d \eta d x .
$$

A computation gives

$$
|\operatorname{deg} g| \leq C \int_{\mathbb{S}^{N}} \int_{\mathbb{S}^{N}} \frac{1}{|\xi(\xi)-g(\eta)|>\delta} \frac{1}{|\xi-\eta|^{2 N}} d \xi d \eta .
$$

Step 2. Proof of assertion (1.3) when $g$ is merely a continuous function from $\mathbb{S}^{N}$ to $\mathbb{S}^{N}$.

The proof is the same as the proof of the Step 2 of [3, Theorem 1.1].

\section{General class of maps from $\mathbb{S}^{N}$ into $\mathbb{S}^{N}$}

In this section, we study the class of $g \in L^{\infty}\left(\mathbb{S}^{N}, \mathbb{S}^{N}\right)$ satisfying (1.6) for some $0<\delta<\ell_{N}$.

5.1 Definition of $\operatorname{deg} g$ when $g \in L^{\infty}\left(\mathbb{S}^{N}, \mathbb{S}^{N}\right)$ satisfies (1.6) for some $0<\delta<\ell_{N}$. We first recall a classical result. 
Lemma 7 (cf. [7, Chapter 4]). Let $g \in L^{\infty}\left(\mathbb{S}^{N}, \mathbb{S}^{N}\right)$ and $\delta \in(0,+\infty)$. Assume that

$$
\int_{\mathbb{S}^{N}} \int_{\substack{\mathbb{S}^{N} \\|g(x)-g(y)|>\delta}} \frac{1}{|x-y|^{2 N}} d x d y<+\infty .
$$

Then

$$
\lim _{r \rightarrow 0} \sup _{x \in \mathbb{S}^{N}} \iint_{\substack{[B(x, r)]^{2} \\|g(\xi)-g(\eta)|>\delta}} \frac{1}{|\xi-\eta|^{2 N}} d \xi d \eta=0 .
$$

Define for each $r>0$,

$$
g_{r}(x)=f_{B(x, r)} g(s) d s, \quad \forall x \in \mathbb{S}^{N} .
$$

The following result is a consequence of Lemmas 6 and 7.

Corollary 5. Let $g \in L^{\infty}\left(\mathbb{S}^{N}, \mathbb{S}^{N}\right)$ and $\delta \in\left(0, \ell_{N}\right)$. Assume that

$$
\int_{\mathbb{S}^{N}} \int_{\mathbb{S}^{N}} \frac{1}{|g(x)-g(y)|>\delta} \underset{|x-y|^{2 N}}{\mid x} d x<+\infty .
$$

Then there exists a positive constant $r_{0}$ such that

$$
\left|g_{r}(x)\right| \geq \frac{1}{128(N+1)}, \quad \forall x \in \mathbb{S}^{N}, \forall 0<r<r_{0},
$$

where $g_{r}$ is defined by (5.1).

Proof. The proof is by contradiction. Suppose that there exists a sequence of positive numbers $\left\{r_{n}\right\}_{n \in \mathbb{N}}$ with $\lim _{n \rightarrow \infty} r_{n}=0$, and a sequence of points $\left\{x_{n}\right\}_{n \in \mathbb{N}} \subset \mathbb{S}^{N}$ such that

$$
\left|g_{r_{n}}\left(x_{n}\right)\right|<\frac{1}{128(N+1)} .
$$

Then by Lemma 6 ,

$$
\liminf _{n \rightarrow \infty} \iint_{\substack{\left[B\left(x_{n}, r_{n}\right)\right]^{2} \\|g(\xi)-g(\eta)|>\delta}} \frac{1}{|\xi-\eta|^{2 N}} d \xi d \eta>0 .
$$

However, this contradicts the fact (see Lemma 7) that

$$
\lim _{r \rightarrow 0} \sup _{x \in \mathbb{S}^{N}} \iint_{\substack{[B(x, r)]^{2} \\|g(\xi)-g(\eta)|>\delta}} \frac{1}{|\xi-\eta|^{2 N}} d \xi d \eta=0 .
$$


We now introduce the following

Definition Let $g \in L^{\infty}\left(\mathbb{S}^{N}, \mathbb{S}^{N}\right)$ be such that

$$
\int_{\mathbb{S}^{N}} \int_{\substack{\mathbb{S}^{N} \\|g(x)-g(y)|>\delta}} \frac{1}{|x-y|^{2 N}} d x d y<+\infty .
$$

Take $0<r<r_{0}$, where $r_{0}$ is the constant in Corollary 5; then $\left|g_{r}(x)\right|>0$ for all $x \in \mathbb{S}^{N}$. Set

$$
\widetilde{g}_{r}(x)=\frac{g_{r}(x)}{\left|g_{r}(x)\right|}, \quad \forall x \in \mathbb{S}^{N},
$$

and define

$$
\operatorname{deg} g=\operatorname{deg} \widetilde{g}_{r}
$$

for any $0<r<r_{0}$.

Since $\widetilde{g}_{r}$ is continuous, $\operatorname{deg} \widetilde{g}_{r}$ is well-defined. Furthermore, using the deformation $\widetilde{g}_{t r+(1-t) r^{\prime}}, 0 \leq t \leq 1$, we have $\operatorname{deg} \widetilde{g}_{r}=\operatorname{deg} \widetilde{g}_{r^{\prime}}$ for every $0<r, r^{\prime}<r_{0}$. Thus $\operatorname{deg} g$ is well-defined by (5.3). This definition in the case $g \in \operatorname{VMO}\left(\mathbb{S}^{N}, \mathbb{S}^{N}\right)$ was presented in [5]. A survey of degree theory for maps in $V M O\left(\mathbb{S}^{N}, \mathbb{S}^{N}\right)$ can be also found there.

5.2 Proof of Inequality (1.7). Take $r=r_{0} / 2$ and $\alpha \leq 1 / 4$ ( $\alpha$ depends only on $N$ ) sufficiently small to be defined later. Here $r_{0}$ denotes the constant in Corollary 5. Consider $\widetilde{g}_{r, \alpha} \in C^{1}\left(\mathbb{S}^{N}, \mathbb{S}^{N}\right)$ such that $\operatorname{deg} \widetilde{g}_{r, \alpha}=\operatorname{deg} \widetilde{g}_{r}$ and $\left\|\widetilde{g}_{r, \alpha}-\widetilde{g}_{r}\right\|_{L^{\infty}\left(\mathbb{S}^{N}\right)} \leq \alpha$, where $\widetilde{g}_{r}$ is defined by (5.2). As in the definition of $u$ and $\rho$ with respect to the map $g$ in Step 1 of the proof of assertion (1.8), we define $u$ and $\rho$ for the map $\widetilde{g}_{r, \alpha}$ (for notational ease).

Recall (see [3]) that

$$
\left|\operatorname{deg} \widetilde{g}_{r, \alpha}\right| \leq \frac{C}{\alpha^{N+1}|\mathcal{B}|} \int_{\rho(x)<1} \frac{d x}{\rho(x)^{N}}
$$

for some $C=C(N)$.

Fix $x \in \mathbb{S}^{N}$ such that $\rho(x)<1$. Then from the definition of $\rho$,

$$
\left|f_{B(x, 2 \rho(x))} \widetilde{g}_{r, \alpha}(y) d y\right|=\alpha .
$$

Since $\left\|\widetilde{g}_{r, \alpha}-\widetilde{g}_{r}\right\|_{L^{\infty}\left(\mathbb{S}^{N}\right)} \leq \alpha$, it follows from (5.5) that

$$
\left|f_{B(x, 2 \rho(x))} \widetilde{g}_{r}(y) d y\right| \leq \alpha+\alpha=2 \alpha .
$$


Since $\alpha \leq 1 / 4$, this implies

$$
\left|f_{B(x, 2 \rho(x))} \widetilde{g}_{r}(y) d y\right| \leq \frac{1}{2}
$$

On the other hand, by Corollary 5,

$$
\left|g_{r}(y)\right|=\left|f_{B(y, r)} g(s) d s\right| \geq \frac{1}{128(N+1)}, \quad \forall y \in \mathbb{S}^{N} .
$$

Combining (5.2), (5.7), and (5.8) yields

$$
r \lesssim \rho(x)
$$

Since

$$
f_{B(x, 2 \rho(x))} \widetilde{g}_{r}(\xi) d \xi=\frac{1}{|B(x, 2 \rho(x))|} \int_{B(x, 2 \rho(x))} f_{B(\xi, r)} \frac{g(y)}{\left|g_{r}(\xi)\right|} d y d \xi,
$$

it follows by Fubini's theorem that

$$
f_{B(x, 2 \rho(x))} \widetilde{g}_{r}(\xi) d \xi=\frac{1}{|B(x, 2 \rho(x))|} \int_{B(x, 2 \rho(x)+r)} \lambda(y) g(y) d y,
$$

where

$$
\lambda(y)=\frac{1}{|B(x, r)|} \int_{\substack{|\xi-y| \leq r \\|\xi-x| \leq 2 \rho(x)}} \frac{1}{\left|g_{r}(\xi)\right|} d \xi
$$

Combining (5.8), (5.9), and (5.11) yields

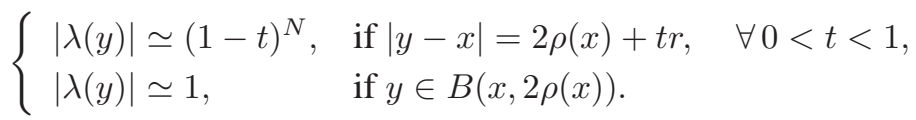

On the other hand, for $0<t<1$, we have from (5.10)

$$
\begin{aligned}
&\left|\int_{B(x, 2 \rho(x)+t r)} \lambda(y) g(y) d y\right| \leq \\
&\left|\int_{B(x, 2 \rho(x))} \widetilde{g}_{r}(\xi) d \xi\right|+\left|\int_{B(x, 2 \rho(x)+r) \backslash B(x, 2 \rho(x)+t r)} \lambda(y) g(y) d y\right| .
\end{aligned}
$$

Thus it follows from (5.6), (5.9), (5.11), and (5.12) that

$$
\left|f_{B(x, 2 \rho(x)+t r)} \lambda(y) g(y) d y\right| \lesssim \alpha+(1-t)^{N+1}, \quad \forall 0<t<1 .
$$


Applying Lemma 6 with $\beta=\sup _{\substack{|y-x|=2 \rho(x)+s r \\ 0<s<t}} \frac{1}{\lambda(y)}\left(\beta \simeq(1-t)^{-N}\right)$ and $\mathcal{D}=$ $B(x, 2 \rho(x)+t r)$, after fixing $t$ sufficiently near to $1(0<t<1)$ and taking $\alpha=(1-t)^{N+1}$, one has

$$
\text { meas }\{(\xi, \eta) \in B(x, 2 \rho(x)+t r):|g(\xi)-g(\eta)|>\delta\} \gtrsim|B(x, 2 \rho(x)+t r)|^{2} .
$$

Thus, just as in the proof of Corollary 5, there exists a positive constant $\tau=\tau(N)$ such that

$$
\underset{\substack{[B(x, 2 \rho(x)+t r)]^{2} \\|g(\xi)-g(\eta)|>\delta \\|\xi-\eta| \geq \tau \rho(x)}}{\int} \frac{1}{|\xi-\eta|^{2 N}} d \xi d \eta \gtrsim 1
$$

On the other hand, from (5.9), there exists a constant $k=k(N)$, depending only on $N$, such that $k \rho(x) \geq 2 \rho(x)+r$. Thus, it follows from (5.13) that

$$
\iint_{\substack{[B(x, k \rho(x))]^{2} \\|g(\xi)-g(\eta)|>\delta \\|\xi-\eta| \geq \tau \rho(x)}} \frac{1}{|\xi-\eta|^{2 N}} d \xi d \eta \gtrsim 1
$$

Combining (5.4) and (5.14) yields

$$
\left|\operatorname{deg} \widetilde{g}_{r, \alpha}\right| \lesssim \int_{\mathbb{S}^{N}} \frac{1}{\rho(x)^{N}} \underset{\substack{[B(x, k \rho(x))]^{2} \\|g(\xi)-g(\eta)|>\delta \\|\xi-\eta| \geq \tau \rho(x)}}{|\xi-\eta|^{2 N}} d \xi d \eta d x
$$

A computation yields

$$
\left|\operatorname{deg} \widetilde{g}_{r, \alpha}\right| \lesssim \int_{\mathbb{S}^{N}} \int_{\mathbb{S}^{N}} \frac{1}{|\xi(\xi)-g(\eta)|>\delta}||^{2 N} d \xi d \eta
$$

Therefore, inequality (1.7) follows, since $\operatorname{deg} g=\operatorname{deg} \widetilde{g}_{r}=\operatorname{deg} \widetilde{g}_{r, \alpha}$.

Acknowledgments. The author is deeply grateful to Prof. H. Brezis for his encouragement. He thanks Prof. H. Brezis for having drawn his attention to this subject and for indicating useful directions. He thanks Prof. P. Mironescu for useful comments and discussions and D. Chiron, R. Ignat, V. Millot and A. Ponce for interesting discussions. 


\section{REFERENCES}

[1] J. Bourgain, H. Brezis and P. Mironescu, Lifting, degree, and distributional Jacobian revisited, Comm. Pure Appl. Math. 58 (2005), 529-551.

[2] J. Bourgain, H. Brezis and P. Mironescu, Complements to the paper: Lifting, Degree, and Distributional Jacobian Revisited, posted on the website http://igd.univ-lyon1.fr/ mironescu/\%5B27C\%5D.pdf.

[3] J. Bourgain, H. Brezis and H-M. Nguyen, A new estimate for the topological degree, C. R. Math. Acad Sci. Paris 340 (2005), 787-791.

[4] H. Brezis, New questions related to the topological degree, in The Unity of Mathematics, Birkhauser Boston, Boston, volume in honor of ninetieth birthday of I. M Gelfand 2006, pp. 137154.

[5] H. Brezis and L. Nirenberg, Degree theory and BMO, Part I: Compact Manifolds without boundaries, Selecta Math. 1 (1995), 197-263.

[6] T. Rockafellar, Convex Analysis, Princeton University Press, Princeton, 1970.

[7] H. L. Royden, Real Analysis, The Macmillan Co., New York, 1963.

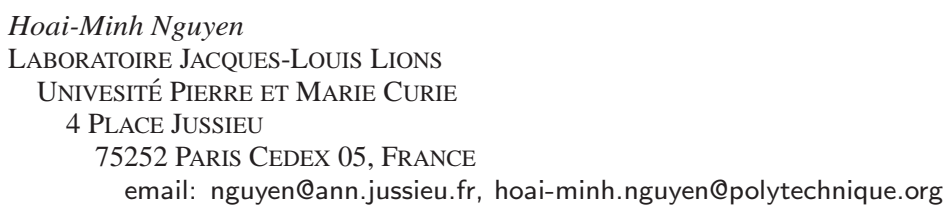

(Received June 19, 2005 and in revised form July 13, 2006) 